\title{
Dynamique saisonnière de l'infestation par les tiques (Ixodoidea) des bovins commercialisés Bayemi P.H. dans la région de Yaoundé, Cameroun
}

BAYEMI (P.H.). Dynamique saisonnière de l'infestation par les tiques (Ixodoidea) des bovins commercialisés dans la région de Yaoundé, Cameroun. Revue Élev. Méd. vét. Pays trop., 1991, 44 (3) : 309-318

Parmi les espèces de tiques identifiées au Cameroun, huit ont été récoltées sur les bovins à Yaoundé : Amblyomma variegatum, Boophilus annulatus, B. decoloratus, Haemaphysalis aciculifer, Hyalomma nitidum, Rhipicephalus longus, $R h$. lunulatus, $R$ h. sulcatus. Trois espèces, $\mathrm{Hm}$. aciculifer, $\mathrm{H}$. nitidum et $\mathrm{Rh}$. lunulatus, ont été identifiées dans cette région pour la première fois. La pullulation de Rhipicephalus et Hyalomma est observée en saison des pluies. Boophilus abonde toute l'année, avec une concentration maximale à la fin de la grande saison sèche. Enfin, Amblyomma, aussi récolté en grande quantité toute l'année, a une infestation minimale en grande saison des pluies. Un seul individu (une femelle) de l'espèce $\mathbf{H m}$. aci. culifer a été trouvée. Mots clés : Tique - Bovin - Localisation Dynamique saisonnière - Cameroun.

\section{INTRODUCTION}

L'amélioration de l'élevage des bovins en Afrique se heurte à plusieurs obstacles parmi lesquels les maladies transmises par les tiques. Nombre d'auteurs ont évalué les dommages causés par ces acariens $(1,7)$. II en ressort que la lutte contre les agents pathogènes doit commencer par un contrôle systématique des tiques. Or la difficulté rencontrée dans ce contrôle réside dans le fait que, pour chaque espèce, il est important de connaître sa biologie et son rôle vectoriel (13).

C'est dans cette perspective que des études ont été menées au Cameroun : RAGEAU $(19,20)$ puis MOREL et MOUCHET $(15,16)$ ont identifié les différentes espèces de tiques et leur répartition géographique. Les travaux de MBAH (9) dans le nord du pays et de MERLIN et al. (11) dans le nord-ouest ont permis de déterminer, respectivement, les mortalités du bétail dues aux tiques et leur dynamique saisonnière.

La région de Yaoundé est une zone de forêt équatoriale où il existe un intérêt croissant pour l'élevage de la race bovine Ndama. Peu de données y existent sur les Ixodoidea pouvant entretenir certaines enzooties. Cette étude a pour

1. Institut de recherches zootechniques, BP 1457, Yaoundé, Cameroun.

* Adresse actuelle : University of Reading, Department of Agriculture, No 1 Earley Gate, Reading, RG6 2AT, England.

Reçu le 13.12.1989, accepté le 16.4.1991. objectif la collecte et l'identification des tiques à Yaoundé, leur localisation sur les bovins, leur variation saisonnière et son implication pour la pathologie bovine.

\section{MATÉRIEL ET MÉTHODE}

\section{Localisation}

Yaoundé, qui se situe à une altitude moyenne de $760 \mathrm{~m}$, est comprise entre 11 et $11^{\circ} 13$ de longitude Est et entre $3^{\circ} 50$ et $4^{\circ} 50$ de latitude Nord.

\section{Climat}

Il est de type tropical humide. Les données climatiques enregistrées à la station météorologique de Yaoundé pendant les 12 mois de l'étude montrent un total de $1883 \mathrm{~mm}$ d'eau. Les températures relevées situent la moyenne des minima à $19,3 \pm 0,5^{\circ} \mathrm{C}$ et celle des maxima à $28,5 \pm 1,2{ }^{\circ} \mathrm{C}$ (tabl. I). II y a alternance de quatre saisons : la grande saison sèche (décembre-février), la petite saison des pluies (mars-juin), la petite saison sèche (juillet-août) et la grande saison des pluies (septembrenovembre).

L'humidité relative est plus marquée près du sol et est inversement proportionnelle à l'ensoleillement.

\section{Végétation}

Les environs de Yaoundé sont dominés par des formations secondaires. La forêt est de type semi-décidu. Les endroits humides, qui sont des prairies marécageuses, sont occupés par des raphiales et ptéritophytes (10). Dans la région d'Étoudi, où se trouve le marché à bétail, on note une action concomitante de l'homme et des animaux. II y a une évolution vers les genres herbacées et ligneux des savanes voisines. Ceux-ci sont associés à d'autres genres fourragers tels que Brachiaria, Panicum, Pennisetum, en composition avec Eupatorium odoratum, considérée comme une peste végétale dans la région.

L'humus recouvre généralement les sols, qui sont riches en oxyde et hydroxyde de fer (17). 


\section{P.I. Bayemi}

TABLEAU I Données climatologiques enregistrées de juin 1985 à mai 1986.

\begin{tabular}{|c|c|c|c|c|c|c|c|c|c|c|c|c|c|}
\hline \multicolumn{2}{|r|}{ Mois } & $\mathrm{J}$ & J & A & $S$ & 0 & $\mathrm{~N}$ & $\mathrm{D}$ & $J$ & $\mathrm{~F}$ & $M$ & A & $M$ \\
\hline \multirow{2}{*}{$\begin{array}{c}\mathrm{T} \\
\left({ }^{\circ} \mathrm{C}\right)\end{array}$} & $M$ & 27,3 & 26,5 & 27,2 & 27,7 & 27,5 & 28,9 & 28 & 29,9 & 30,4 & 29,1 & 29,5 & 29,4 \\
\hline & $\mathrm{m}$ & 19,2 & 18,9 & 19 & 18,6 & 18,7 & 19,6 & 19,1 & 19,3 & 20,3 & 19,1 & 20 & 19,5 \\
\hline \multicolumn{2}{|c|}{$\underset{(\mathrm{mm})}{\mathrm{P}}$} & 166,5 & 118,5 & 189,6 & 320,7 & 436,6 & 180,7 & 9,8 & 0 & 38,4 & 126,4 & 155,1 & 141 \\
\hline
\end{tabular}

$T$ : température ; $M:$ maxima ; $m:$ minima ; $P$ : pluviométrie.

\section{Animaux}

Les bovins en vente au marché d'Étoudi à Yaoundé proviennent surtout de l'Adamaoua, de l'Est et, rarement, de Banyo (fig. 1). Les animaux, jeunes et adultes (plus de 18 mois), sont de races locales : Gudali Banyo, Gudali Ngaoundéré, Aku, Bororo ou leurs croisés. Ils n'étaient pas traités contre les tiques au lieu de vente et y ont séjourné pendant plusieurs semaines. En général, un animal n'était examiné qu'une seule fois.

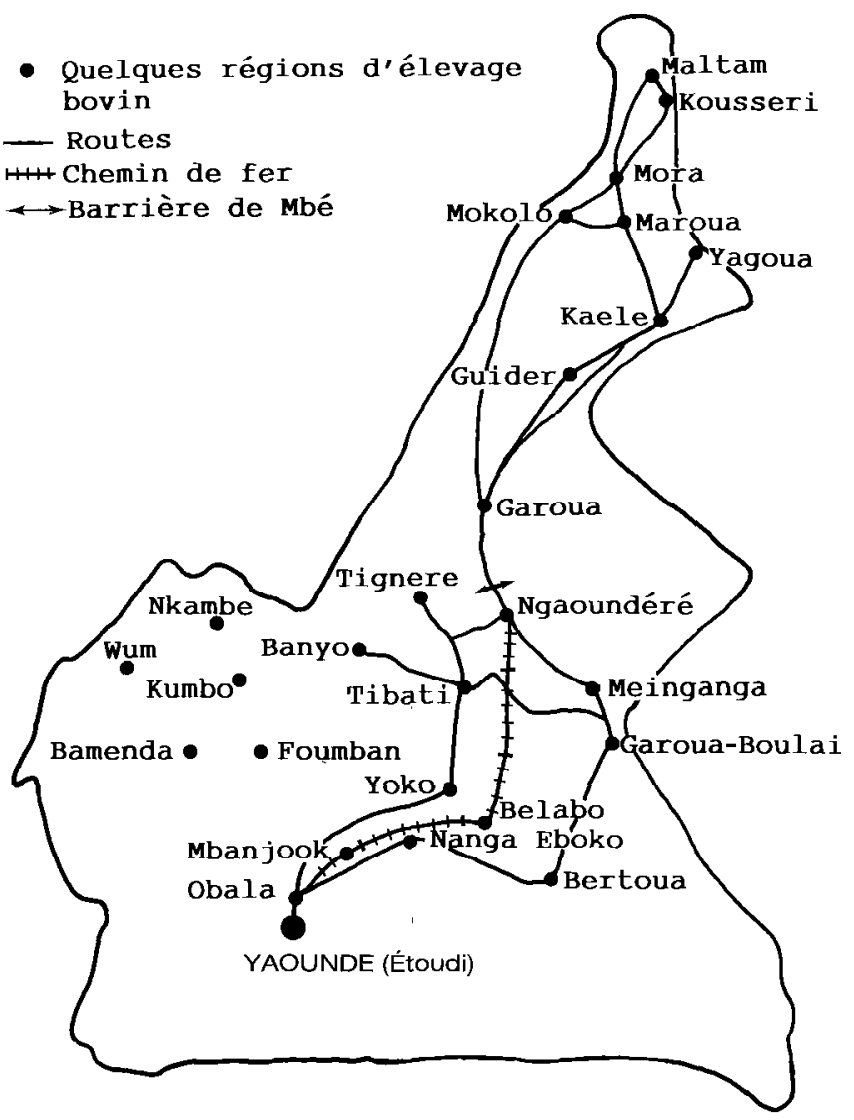

Fig. 1 : Provenance des bovins du marché d'Étoudi.

\section{Technique de récolte}

La récolte se faisait à main nue, une fois tous les 15 jours, de juịn 1985 à mai 1986. Cinq animaux dociles choisis au hasard sont couchés par terre et attachés. Les tiques sont collectées sur différentes parties du corps (fig. 2) et directement introduites dans l'alcool à $70^{\circ}$ pour conservation avant d'être étudiées au laboratoire.

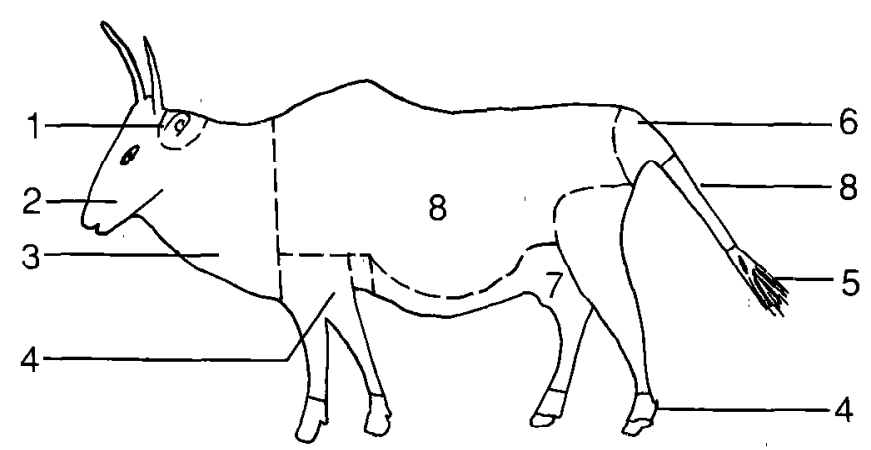

Fig. 2 : Régions anatomiques d'une femelle Gudali $: 1$, oreilles $; 2$, tête $: 3$, cou $; 4$, pattes + aisselles + espaces interdigitaux $; 5$, toupillon $; 6$, anus 7 , testicules + mamelles $; 8$, reste du corps.

\section{Analyses statistiques}

Pour vérifier si d'autres facteurs (compétitions) influencent la dynamique des tiques sur l'hôte, il importe de calculer les corrélations entre les différentes espèces d'un même genre et entre les divers genres. Ce calcul a été fait sur le programme d'ordinateur MSTAT (17).

L'indice quotidien moyen (IQM) a été calculé selon la formule suivante : $I Q M=N / D$, où $N$ est le nombre de tiques récoltées lors d'un prélèvement par hôte selon l'espèce et la stase, D la durée moyenne de fixation sur l'hôte selon l'espèce et la stase, exprimée en jours.

Cet indice représente le nombre estimé de tiques d'une espèce et d'une stase données, qui sont supposées avoir terminé leur repas de sang un jour donné (2). 
Les temps moyens de gorgement (2) ont été pris comme suit : A. variegatum : larves, 8,2 jours ; nymphes, 6,3 jours ; femelles, 13,5 jours ; mâles, 50 jours ; Boophilus : séjour complet des femelles, 21 jours ; Rh. lunulatus : femelles, 13 jours ; mâles, 40 jours (ces temps ont été appliqués aux autres espèces de Rhipicephalus) ; $H$. nitidum : femelles, 6 jours ; mâles, 30 jours.

\section{RÉSULTATS}

Les tableaux II à VI représentent des collectes annuelles faites sur dix animaux. Les tableaux VII à $X$ contiennent les résultats des collectes mensuelles sur dix animaux. Seul le tableau XI présente l'indice quotidien moyen par animal.

\section{Amblyomma variegatum}

Les larves ont été récoltées 10 mois sur 12 , de juin à mars, avec trois pics : juin, septembre et décembre. Plus de 50 p. 100 de ces larves sont trouvées à la tête et aúx oreilles.

Les nymphes sont prédominantes aux pattes et aux parties génitales. Leur activité s'étend de septembre à mai ; elle est plus marquée en grande saison sèche (novembre à mars).

Quant aux imagos, plus de 75 p. 100 se localisent aux pattes, aux testicules et aux mamelles. Ils sont présents toute l'année.

\section{Boophilus}

Ce genre représente 77 p. 100 du total des tiques récoltées (tabl. III). II peut se gorger sur toutes les parties de l'animal sauf au toupillon.

TABLEAU II Localisation de Amblyomma variegatum.

\begin{tabular}{|c|c|c|c|c|c|c|c|c|c|c|c|}
\hline \multicolumn{2}{|c|}{ Localisation } & Tête & Cou & Oreilles & $\begin{array}{c}\text { Pattes } \\
+ \\
\text { aisselles }\end{array}$ & Queue & Anus & $\begin{array}{c}\text { Testicules } \\
+ \\
\text { mamelles }\end{array}$ & $\begin{array}{l}\text { Reste } \\
\text { du } \\
\text { corps }\end{array}$ & Total & p. 100 \\
\hline \multicolumn{2}{|l|}{ Larves } & 20 & 19 & 54 & 7 & 1 & 4 & 1 & 6 & 112 & \multirow{5}{*}{18} \\
\hline \multicolumn{2}{|l|}{ Nymphes } & 7 & 55 & 22 & 269 & 28 & 4 & 213 & 47 & 645 & \\
\hline \multirow{2}{*}{ A. variegatum } & $\sigma$ & 59 & 44 & 18 & 469 & 1 & 102 & 565 & 86 & 1346 & \\
\hline & 우 & 27 & 14 & 15 & 208 & 7 & 53 & 274 & 44 & 642 & \\
\hline \multicolumn{2}{|l|}{ Total } & 113 & 132 & 109 & 953 & 37 & 163 & 1053 & 185 & 2745 & \\
\hline
\end{tabular}

む: mâles ; o : femelles.

TABLEAU III Localisation de Boophilus.

\begin{tabular}{|c|c|c|c|c|c|c|c|c|c|c|c|}
\hline \multicolumn{2}{|c|}{ Localisation } & Tête & Cou & Oreilles & $\begin{array}{c}\text { Pattes } \\
+ \\
\text { aisselles }\end{array}$ & Queue & Anus & $\begin{array}{c}\text { Testicules } \\
+ \\
\text { mamelles }\end{array}$ & $\begin{array}{c}\text { Reste } \\
\text { du } \\
\text { corps }\end{array}$ & Total & p. 100 \\
\hline \multicolumn{2}{|l|}{ Larves } & 24 & 8 & 35 & 5 & - & 29 & 21 & 9 & 131 & \multirow{7}{*}{77,5} \\
\hline \multicolumn{2}{|l|}{ Nymphes } & 87 & 128 & 60 & 107 & - & 90 & 189 & 134 & 795 & \\
\hline \multirow{2}{*}{ B. annulatus } & 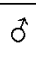 & 2 & 8 & 2 & 37 & - & 11 & 79 & 3 & 142 & \\
\hline & $\varphi$ & 44 & 24 & 6 & 183 & - & 22 & 217 & 44 & 540 & \\
\hline \multirow{2}{*}{ B. decoloratus } & $\hat{o}$ & 120 & 288 & 48 & 382 & - & 173 & 614 & 595 & 2220 & \\
\hline & q & 258 & 968 & 182 & 1903 & 1 & 614 & 1737 & 2213 & 7876 & \\
\hline \multicolumn{2}{|l|}{ Total } & 535 & 1424 & 333 & 2517 & 1 & 959 & 2857 & 2998 & 11704 & \\
\hline
\end{tabular}




\section{P.H. Bayemi}

TABLEAUIV Localisation de Rhipicephalus.

\begin{tabular}{|c|c|c|c|c|c|c|c|c|c|c|c|}
\hline \multicolumn{2}{|c|}{ Localisation } & Tête & Cou & Oreilles & $\begin{array}{c}\text { Pattes } \\
+ \\
\text { aisselles }\end{array}$ & Queue & Anus & $\begin{array}{c}\text { Testicules } \\
+ \\
\text { mamelles }\end{array}$ & $\begin{array}{c}\text { Reste } \\
\text { du } \\
\text { corps }\end{array}$ & Total & p. 100 \\
\hline \multicolumn{2}{|l|}{ Larves } & - & - & - & - & - & - & - & - & - & \multirow{9}{*}{3,5} \\
\hline \multicolumn{2}{|l|}{ Nymphes } & - & - & - & - & - & - & - & - & - & \\
\hline \multirow{2}{*}{ Rh. sulcatus } & $\delta$ & - & - & 55 & - & 17 & - & - & - & 72 & \\
\hline & 우 & - & - & 24 & - & 1 & - & - & 1 & 26 & \\
\hline \multirow{2}{*}{ Rh. lunulatus } & $\sigma^{\pi}$ & 2 & - & 1 & 3 & 240 & 2 & 2 & 3 & 253 & \\
\hline & q & - & - & - & 1 & 131 & 3 & - & 1 & 136 & \\
\hline \multirow{2}{*}{ Rh. longus } & 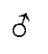 & 1 & 2 & 3 & 10 & 3 & - & 6 & 2 & 27 & \\
\hline & q & - & - & - & 2 & 1 & - & 1 & - & 2 & \\
\hline \multicolumn{2}{|l|}{ Total } & 3 & 2 & 83 & 16 & 393 & 5 & 9 & 7 & 518 & \\
\hline
\end{tabular}

ઉ: mâles ; ㅇ : femelles.

TABLEAUV Localisation de Hyalomma nitidum.

\begin{tabular}{|c|c|c|c|c|c|c|c|c|c|c|c|}
\hline \multicolumn{2}{|c|}{ Localisation } & Tête & Cou & Oreilles & $\begin{array}{c}\text { Pattes } \\
+ \\
\text { aisselles }\end{array}$ & Queue & Anus & $\begin{array}{c}\text { Testicules } \\
+ \\
\text { mamelles }\end{array}$ & $\begin{array}{c}\text { Reste } \\
\text { du } \\
\text { corps }\end{array}$ & Total & p. 100 \\
\hline \multicolumn{2}{|l|}{ Larves } & - & - & - & - & - & - & - & - & - & \\
\hline \multicolumn{2}{|l|}{ Nymphes } & - & - & - & - & - & - & - & - & - & \\
\hline \multirow{2}{*}{ H. nitidum } & 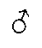 & - & 3 & - & 34 & 35 & - & 44 & 9 & 128 & 1 \\
\hline & $q$ & - & - & - & 2 & 11 & - & 7 & 2 & 22 & \\
\hline \multicolumn{2}{|l|}{ Total } & - & 3 & - & 36 & 46 & - & 51 & 11 & 150 & \\
\hline
\end{tabular}

đ: mâles ; $:$ : femelles.

TABLEAU VI Récapitulatif par région anatomique.

\begin{tabular}{|c|c|c|c|c|c|c|c|c|c|c|}
\hline Localisation & Tête & Cou & Oreilles & $\begin{array}{c}\text { Pattes } \\
+ \\
\text { aisselles }\end{array}$ & Queue & Anus & $\begin{array}{c}\text { Testicules } \\
+ \\
\text { mamelles }\end{array}$ & $\begin{array}{c}\text { Reste } \\
\text { du } \\
\text { corps }\end{array}$ & Total & p. 100 \\
\hline Grand total & 691 & 1561 & 525 & 3622 & 477 & 1110 & 3970 & 3201 & 15117 & \\
\hline Pourcentage & 4.31 & 10,33 & 3,47 & 23,96 & 3,16 & 7,34 & 26,26 & 21,17 & 100 & 100 \\
\hline
\end{tabular}


TABLEAU VII Variation saisonnière de Amblyomma variegatum.

\begin{tabular}{|c|c|c|c|c|c|c|c|c|c|c|c|c|c|}
\hline \multicolumn{2}{|l|}{ Mois } & J & $\mathrm{J}$ & A & $S$ & 0 & $\mathrm{~N}$ & D & $J$ & $\mathrm{~F}$ & $M$ & A & $M$ \\
\hline \multicolumn{2}{|l|}{ Larves } & 14 & 8 & 0 & 42 & 7 & 7 & 17 & 11 & 5 & 1 & 0 & 0 \\
\hline \multicolumn{2}{|l|}{ Nymphes } & 5 & 4 & 6 & 52 & 19 & 105 & 111 & 110 & 101 & 75 & 41 & 16 \\
\hline \multirow{2}{*}{ A. variegatum } & $\sigma^{\pi}$ & 86 & 93 & 117 & 34 & 31 & 28 & 19 & 54 & 129 & 322 & 303 & 130 \\
\hline & $q$ & 82 & 56 & 86 & 28 & 12 & 10 & 6 & 21 & 43 & 85 & 133 & 80 \\
\hline \multicolumn{2}{|l|}{ Sex-ratio } & 1,05 & 1,66 & 1,36 & 1,21 & 2,58 & 2,80 & 3,16 & 2,57 & 3,00 & 3,79 & 2,28 & 1,62 \\
\hline
\end{tabular}

ઠ̊: mâles ; ㅇ : femelles.

TABIEAU VIII Variation saisonnière de Boophilus.

\begin{tabular}{|c|c|c|c|c|c|c|c|c|c|c|c|c|c|}
\hline \multicolumn{2}{|l|}{ Mois } & $J$ & $\mathrm{~J}$ & A & $S$ & 0 & $N$ & $\mathrm{D}$ & J & $F$ & M & A & $M$ \\
\hline \multicolumn{2}{|l|}{ Larves } & 12 & 9 & 33 & 0 & 2 & 9 & 5 & 29 & 21 & 10 & 1 & 0 \\
\hline Nymphes & & 33 & 66 & 171 & 109 & 33 & 85 & 84 & 72 & 76 & 32 & 20 & 14 \\
\hline \multirow{2}{*}{ B. annulatus } & $\hat{o}$ & 6 & 9 & 32 & 20 & 1 & 9 & 12 & 15 & 15 & 11 & 8 & 4 \\
\hline & $q$ & 10 & 109 & 61 & 144 & 11 & 16 & 28 & 40 & 39 & 38 & 30 & 14 \\
\hline \multirow{2}{*}{ B. decoloratus } & o & 42 & 281 & 196 & 244 & 98 & 114 & 291 & 391 & 274 & 119 & 66 & 104 \\
\hline & $q$ & 323 & 581 & 773 & 844 & 414 & 433 & 859 & 1115 & 1131 & 686 & 317 & 394 \\
\hline
\end{tabular}

ઉ: mâles ; 9 : femelles.

TABLEAU IX Variation saisonnière de Rhipicephalus.

\begin{tabular}{|c|c|c|c|c|c|c|c|c|c|c|c|c|c|}
\hline \multicolumn{2}{|c|}{ Mois } & $J$ & J & A & $S$ & $\mathrm{O}$ & $\mathrm{N}$ & $D$ & $J$ & $\mathrm{~F}$ & $M$ & A & $M$ \\
\hline \multicolumn{2}{|l|}{ Larves } & - & - & - & - & - & 一 & 一 & - & 一 & - & - & - \\
\hline \multicolumn{2}{|l|}{ Nymphes } & - & - & - & - & - & - & - & - & - & - & - & - \\
\hline \multirow{2}{*}{ Rh. longus } & $\hat{\sigma}$ & 2 & 2 & 1 & 4 & 6 & 1 & 0 & 1 & 5 & 4 & 1 & 0 \\
\hline & $q$ & 1 & 0 & 2 & 0 & 1 & 0 & 0 & 0 & 0 & 0 & 0 & 0 \\
\hline \multirow{2}{*}{ Rh. lunulatus } & $\delta$ & 41 & 72 & 69 & 8 & 2 & 0 & 0 & 0 & 0 & 1 & 20 & 40 \\
\hline & q & 23 & 45 & 28 & 28 & 9 & 0 & 0 & 0 & 0 & 0 & 1 & 2 \\
\hline \multirow{2}{*}{ Rh. sulcatus } & 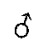 & 9 & 41 & 22 & 0 & 0 & 0 & 0 & 0 & 0 & 0 & 0 & 0 \\
\hline & $q$ & 5 & 19 & 0 & 0 & 2 & 0 & 0 & 0 & 0 & 0 & 0 & 0 \\
\hline
\end{tabular}


P.H. Bayemi

TABLEAU X Variation saisonnière de Hyalomma nitidum.

\begin{tabular}{|c|c|c|c|c|c|c|c|c|c|c|c|c|c|}
\hline \multicolumn{2}{|c|}{ Mois } & $J$ & $J$ & $A$ & $S$ & 0 & $\mathrm{~N}$ & $D$ & J & $F$ & $M$ & $A$ & $M$ \\
\hline \multicolumn{2}{|l|}{ Larves } & - & - & - & - & - & - & - & 一 & - & - & - & - \\
\hline \multicolumn{2}{|l|}{ Nymphes } & - & - & - & - & - & - & - & 一 & - & - & 一 & - \\
\hline \multirow{2}{*}{ H. nitidum } & $\sigma^{*}$ & 3 & 16 & 18 & 50 & 11 & 10 & 7 & 2 & 5 & 5 & 1 & 0. \\
\hline & 우 & 0 & 2 & 4 & 8 & 4 & 1 & 2 & 1 & 0 & 0 & 0 & 0 \\
\hline
\end{tabular}

ठ̊: mâles ; ㅇ : femelles.

TABLEAU XI Variation de l'indice quotidien moyen (IQM) d'infestation.

\begin{tabular}{|c|c|c|c|c|c|c|c|c|c|c|c|c|c|}
\hline \multicolumn{2}{|l|}{ Mois } & $J$ & $J$ & $A$ & $\mathrm{~S}$ & 0 & $N$ & $D$ & $\mathrm{~J}$ & $F$ & M & A & M \\
\hline A. variegatum & $\begin{array}{l}\mathrm{L} \\
\mathrm{N} \\
0 \\
0 \\
+\end{array}$ & $\begin{array}{l}0,17 \\
0,08 \\
0,17 \\
0,6\end{array}$ & $\begin{array}{l}0,1 \\
0,06 \\
0,18 \\
0,4\end{array}$ & $\begin{array}{l}0 \\
0,1 \\
0,2 \\
0,63\end{array}$ & $\begin{array}{l}0,5 \\
0,82 \\
0,068 \\
0,2\end{array}$ & $\begin{array}{l}0,08 \\
0,03 \\
0,062 \\
0,08\end{array}$ & $\begin{array}{l}0,08 \\
1,67 \\
0,05 \\
0,7\end{array}$ & $\begin{array}{l}0,2 \\
1,76 \\
0,038 \\
0,04\end{array}$ & $\begin{array}{l}0,13 \\
1,74 \\
0,1 \\
0,15\end{array}$ & $\begin{array}{l}0,06 \\
1,6 \\
0,25 \\
0,3\end{array}$ & $\begin{array}{l}0,012 \\
1,2 \\
0,64 \\
0,63\end{array}$ & $\begin{array}{l}0 \\
0,6 \\
0,6 \\
0,98\end{array}$ & $\begin{array}{l}0 \\
0,2 \\
0,26 \\
0,59\end{array}$ \\
\hline B. annulatus & q & 0,047 & 0,52 & 0,29 & 0,68 & 0,05 & 0,07 & 0,13 & 0,19 & 0,18 & 0,06 & 0,14 & 0,06 \\
\hline B. decoloratus & q & 1,5 & 2,7 & 3,6 & 4 & 1,9 & 2 & 4 & 5,31 & 5,38 & 3,24 & 1,51 & 1,87 \\
\hline Rh. longus & 우 & 0,007 & 0 & 0,015 & 0 & 0,007 & 0 & 0 & 0 & 0 & 0 & 0 & 0 \\
\hline \multirow{2}{*}{ Rh. lunulatus } & ๙ & 0,1 & 0,18 & 0,17 & 0,02 & 0,005 & 0 & 0 & 0 & 0 & 0,002 & 0,05 & 0,1 \\
\hline & q & 0,17 & 0,34 & 0,21 & 0,21 & 0,07 & 0 & 0 & 0 & 0 & 0 & 0,007 & 0,015 \\
\hline \multirow{2}{*}{ Rh. sulcatus } & $\sigma^{\star}$ & 0,02 & 0,1 & 0,05 & 0 & 0 & 0 & 0 & 0 & 0 & 0 & 0 & 0 \\
\hline & 우 & 0,03 & 0,14 & 0 & 0 & 0,015 & 0 & 0 & 0 & 0 & 0 & 0 & 0 \\
\hline \multirow{2}{*}{ H. nitidum } & 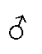 & 0,01 & 0,05 & 0,06 & 0,16 & 0,036 & 0,03 & 0,02 & 0,006 & 0,16 & 0,16 & 0,003 & 0 \\
\hline & q & 0 & 0,03 & 0,06 & 0,13 & 0,06 & 0,016 & 0,03 & 0,016 & 0 & 0 & 0 & 0 \\
\hline
\end{tabular}

$L$ : larves ; $N$ : nymphes ; ‘̃ : mâles; 9 : femelles.

\section{B. annulatus}

Cette espèce préfère les pattes et les parties génitales.

Sa phase parasitaire s'étend sur toute l'année.

\section{B. decoloratus}

Elle suit la courbe des variations de $B$. annulatus, mais à une plus grande échelle. En plus des deux sites précités, elle se localise au tronc.

\section{Rhipicephalus}

Seuls les imagos étaient présents sur les bovins.
Rh. longus

Quelques individus seulement ont été récoltés sur tout le corps avec 40 p. 100 de préférence pour les pattes.

Rh. lunulatus

Son activité sur l'hôte définitif est presque nulle en grande saison sèche. Mais elle réapparaît dès l'arrivée des pluies et atteint son plus haut niveau d'infestation en juillet. Ele n'est pas rencontrée au cou, mais 95 p. 100 se localisent au toupillon. 


\section{Rh. sulcatus}

Elle réside surtout aux oreilles et accessoirement au toupillon. Le parasitisme des adultes se limite à la petite saison sèche (juin-août). Le reste de l'année, elle se fait plus rare.

\section{Hyalomma nitidum}

Ne se rencontre pas à la tête et aux oreilles, mais à plus de 34 p. 100 aux parties génitales, 30 p. 100 à la queue et 24 p. 100 aux pattes (tabl. V). Sa présence varie proportionnellement à la pluviométrie.

\section{Haemaphysalis}

Un seul individu a été trouvé aux pattes ( $H \mathrm{~m}$. aciculifer femelle).

\section{DISCUSSION}

Deux espèces animales sont en compétition dans un biotope si, du fait de la concurrence, une espèce disparaît. Ici, il n'y pas de compétition entre les huit espèces de tiques prélevées. Cette hypothèse a été vérifiée par des tests statistiques en calculant les corrélations entre les différentes espèces d'un même genre et entre les divers genres (tabl. XII et XIII).

Ces analyses montrent qu'il n'y a ni compétition entre ces différents groupes, ni même corrélation, sauf pour $R$. sulcatus se localisant à la conque auriculaire et $R$. lunulatus se gorgeant au toupillon ( $r=0,86 ; P<0,1$ p. 100). Cette étude ayant été menée sur des animaux pâturant dans

TABLEAU XII Analyse de corrélation entre les espèces de Rhipicephalus et celles de Boophilus.

\begin{tabular}{|l|c|c|}
\hline \multicolumn{1}{|c|}{ Espèces } & Corrélation & Probabilité \\
\hline $\begin{array}{l}\text { Rh. longus } \\
\text { x } \\
\text { Rh. lunulatus }\end{array}$ & $-0,044$ & 1 \\
\hline $\begin{array}{l}\text { Rh. longus } \\
\times\end{array}$ & $-0,027$ & 1 \\
$\begin{array}{l}\text { Rh. sulcatus } \\
\begin{array}{l}\text { Rh. lunulatus } \\
\times\end{array}\end{array}$ & 0,866 \\
$\begin{array}{l}\text { Rh. sulcatus } \\
\begin{array}{l}\text { B. annulatus } \\
\mathrm{x}\end{array}\end{array}$ & 0,454 \\
\hline \begin{tabular}{l} 
B. decoloratus \\
\hline
\end{tabular}
\end{tabular}

TABLEAU XIII Analyse de corrélation entre les genres.

\begin{tabular}{|c|c|c|c|}
\hline Genres & Corrélation & \multicolumn{2}{|l|}{ Probabilité } \\
\hline $\begin{array}{l}\text { Amblyomma } \\
x \\
\text { Boophilus }\end{array}$ & $-0,145$ & 1 & \\
\hline $\begin{array}{l}\text { Amblyomma } \\
\mathrm{x} \\
\text { Rhipicephalus }\end{array}$ & $-0,217$ & 1 & \\
\hline $\begin{array}{l}\text { Amblyomma } \\
x \\
\text { Hyalomma }\end{array}$ & $-0,370$ & 0,236 & \\
\hline $\begin{array}{l}\text { Boophilus } \\
\quad x \\
\text { Rhipicephalus }\end{array}$ & 0,049 & 1 & \\
\hline $\begin{array}{l}\text { Boophilus } \\
x \\
\text { Hyalomma }\end{array}$ & 0,340 & 0,279 & 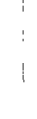 \\
\hline $\begin{array}{l}\text { Rhipicephalus } \\
\times \\
\text { Hyalomma }\end{array}$ & 0,239 & 1 & \\
\hline
\end{tabular}

une même zone, l'interprétation des résultats vis-à-vis de la dynamique des tiques ne sera basée que sur les saisons.

\section{Amblyomma variegatum}

Du point de vue numérique, cette espèce vient en deuxième position (5) : environ 18 p. 100 . Alors que dans le nord-ouest du Cameroun MERLIN et al. (11) ne récoltent pas les larves sur les bovins, celles-ci le sont à Yaoundé, où la présence des petits mammifères sauvages et des oiseaux, hôtes préférés des préimagos (18), est diminuée du fait de l'urbanisation.

Les nymphes pullulent en grande saison sèche $(11,15)$. En grande saison des pluies, les imagos passent leur période parasitaire la moins forte. Ils apparaissent vers la fin de la saison sèche, les mâles en premier. Le sex ratio, nombre de mâles pour une femelle, atteint son maximum, les femelles viennent ensuite (6). En juin, le sex ratio est sensiblement égal à un. C'est une période de copulation intense, suivie par une phase de ponte (juillet-août), une phase larvaire (septembre) et une phase nymphale (novembre-mars).

II y a donc une seule génération par an. STRICKLAND et al. (21) qui ont évalué le cycle minimal de $A$. variegatum à 141 jours, pensent que dans les régions à deux saisons des pluies cette tique peut présenter deux générations par an. Ce n'est pas le cas ici à cause des phases à jeun (parfois très longues) passées au sol. 
Du point de vue pathologique, une seule nymphe, ou un adulte, infectée et gorgée est capable de transmettre la cowdriose (3). Cette maladie peut être dépistée pendant toute l'année. La pullulation des adultes en mars-avril entraîne des riques de dermatophilose.

\section{Boophilus}

Les courbes de variation des deux espèces $B$. decoloratus et $B$. annulatus peuvent se superposer. Quoique $B$. annulatus soit moins abondante, ces deux tiques sont sensibles, de manière synchrone, aux facteurs les affectant. $B$. annulatus ne profite pas de la baisse de l'incidence de $B$. decoloratus pour prendre sa place sur l'hôte (8). L'accroissement de l'infestation des Boophilus aux saisons sèches (21) peut être dû au fait que la période d'oviposition se réduit avec l'augmentation de la température (4) ; la durée du cycle est donc réduite et il y a pullulation des adultes. D'autre part, en fin de saison sèche, l'affaiblissement des animaux diminue leur résistance aux tiques, qui vont se fixer en grand nombre et se gorger plus rapidement (11).

Le genre Boophilus est vecteur de babésiose et d'anaplasmose. II est présent toute l'année et ces maladies peuvent donc être rencontrées à tout moment. Cependant, seul $B$. annulatus transmet facilement la babésiose, mais son petit nombre (moins de 6 à 8 femelles gorgées par animal) ne permet pas l'établissement d'une prémunition précoce et donc une situation endémique stable (22).

\section{Rhipicephalus}

Rh. longus est collectée en nombre très réduit. En Éthiopie, il faut aussi une pluviométrie supérieure à 1200 $\mathrm{mm}$ pour qu'elle infeste normalement les hôtes définitifs (18).

L'infestation par $R h$. lunulatus est optimale en petite saison sèche. Au Nigeria, 50 p. 100 de cette espèce se limite à la saison des pluies (12). A Yaoundé, comme dans la province de Shoa, en Éthiopie, sa période de pullulation est plus longue que celle des autres espèces (18).

$R h$. sulcatus se limite aussi à la petite saison sèche. Dans le nord-ouest du Cameroun, elle parasite les bovins pendant toute la saison des pluies (11)

\section{Hyalomma nitidum}

La phase parasitaire des adultes se situe entre août et octobre. Le pic est atteint en septembre. Au Nigeria, les imagos sont collectés en mai et août (12). C'est la seule espèce qui ne figurait pas dans les travaux de MOREL et MOUCHET en 1965 (16); car son identité n'était pas reconnue à l'époque.

\section{Haemaphysalis aciculifer}

Un seul individu, dont l'identification a été confirmée par P.C. MOREL sous le couvert de P. MERLIN, a été identifié. C'est une espèce vivant en climat humide, dans les fourrés (14), et se gorgeant surtout sur les ongulés sauvages. Elle peut être contractée par les bovins aux abords des ruisseaux.

\section{CONCLUSION}

Cinq genres d'Amblyommidae ont été trouvés au marché de bovins de Yaoundé : Amblyomma, Boophilus, Rhipicephalus, Hyalomma et Haemaphysalis. Trois des huit espèces correspondant à ces cinq genres n'avaient pas encore été identifiées dans cette zone $(15,16,19$, 20). II s'agit de Rhipicephalus lunulatus, Hyalomma nitidum et Haemaphysalis aciculifer. Les deux premières ont été probablement introduites dans la région par l'importation régulière de bétail du nord du pays, puisque ce sont des tiques de savanes et de steppes sud-soudanéennes (14). Or, $R$. evertsi, supposée abondante dans l'Adamaoua, n'a pas été prélevée. C'est aussi une espèce de savane et de steppe dont l'habitat est situé dans des régions où la pluviométrie annuelle est comprise entre 400 et $1000 \mathrm{~mm}$ d'eau. II est donc possible que cette tique ne s'adapte pas au climat de la région de Yaoundé, ce qui reste à vérifier par élevage dans des cages en milieu naturel.

Pour toutes les espèces de tiques rencontrées, les sites préférentiels des mâles sont toujours les mêmes que pour les femelles. Plus de 50 p. 100 de la charge parasitaire se localise aux pattes et aux parties génitales.

Puisque Boophilus annulatus et Amblyomma variegatum parasitent les bovins pendant toute l'année, les babésioses, l'anaplasmose et la cowdriose peuvent être dépistées à toute saison.

Pour la suite de cette étude, à Yaoundé, les étapes suivantes sont envisagées : la recherche de la durée des diverses stases de développement des espèces de tiques rencontrées, la fréquence des maladies transmises ainsi que les méthodes de lutte contre ces Ixodoidea.

\section{REMERCIEMENTS}

Je voudrais exprimer ma gratitude au Dr Paul MERLIN sans qui ce travail n'aurait pu être réalisé. Ma reconnaissance va également au Dr Tarounga BERAMGOTO, McHUGH et Léonard BONONO. 
BAYEMI (P.H.). Seasonal dynamics of tick infestations (Ixodoidea) in cattle in the area of Yaoundé, Cameroon. Revue Élev. Méd. vét. Pays trop., 1991, 44 (3) : 309-318

Among the tick species identified in Cameroon, eight were found in cattle in Yaoundé : Amblyomma variegatum, Boophilus annulatus, B. decoloratus, Haemaphysalis aciculifer, Hyalomma nitidum, Rhipicephalus longus, Rh. lunulatus, Rh. sulcatus. Three species, $\mathbf{H m}$. aciculifer, $\boldsymbol{H}$. nitidum and $\boldsymbol{R} h$. lunulatus, were identified for the first time in Yaoundé. Rhipicephalus and Hyalomma were abundent during the rainy season. Boophilus was abundent throughout the year, with a maximum concentration at the end of the long dry sea. son. Amblyomma, which was encountered in large quantities all year round, had a minimum infestation rate during the long rainy season. Only one female tick of $\mathbf{H m}$. aciculifer was collected. Key words : Tick - Cattle - Localization - Seasonal dynamic - Cameroon.
BAYEMI (P.H.). Dinámica estacional de la infestación por garrapatas (Ixodoidea) de los bovinos comercializados en el área de Yaundé (Camerún). Revue Élev. Méd. vét. Pays trop., 1991, 44 (3) : 309-318

Entre las especies de garrapatas identificadas en el Camerún, ocho fueron colectadas en bovinos en Yaundé : Amblyomma variegatum, Boophilus annulatus, B. decoloratus, Haemaphysalis aciculifer, Hyalomma nitidum, Rhipicephalus longus, $R$ h. lunulatus, $R h$. sulcatus. Tres especies, $\mathrm{Hm}$. aciculifer, $\boldsymbol{H}$. nitidum y $R$. lunulatus, fueron encontrados en la zona por la primera vez. La pululación de Rhipicephalus y Hyalomma se observe durante la estación lluviosa. Boophilus abunda todo el año, con máxima concentración al fín de la gran estación seca. En fín Amblyomma, tambien colectados en gran cuantitad a lo largo del año, presenta su mínima infestación durante la gran estación de lluvias. Una única hembra de $\mathbf{H m}$. aciculifer fué encontrada. Palabras claves : Garrapata - Bovino - Localización Dinámica estacional - Camerún.

\section{BIBLIOGRAPHIE}

1 AJAYI (S.A.), FABIYI (J.I.), UMO (I.). Clinical bovine anaplasmosis and babesiosis in Friesian cattle. An outbreak in Nigeria and its control. Wrld Anim. Rev., 1982, $43: 41$.

2. BARRÉ (N.), CAMUS (E.). Étude épidémiologique de la cowdriose aux Antilles. IEMVT, Mission Antilles-Guyane, 1983. Rapport annuel 1983 .

3. CAMUS (E.), BARRÉ (N.). La cowdriose. Revue générale des connaissances. Maisons-Alfort, IEMVT, 1982 (Études et synthèses, $\left.\mathrm{n}^{\circ} 4\right)$.

4. CAUTAM (O.P.). Cycle de vie de Boophilus amulatus. Indian J. Anim. Sci., 1982, 52 (7). 502 p.

5. GUEYE (A.), MBENGUE (M.), DIOUF (A.), SEYE (M.). Tiques et hémoparasitoses du bétail au Sénégal. La région des Niayes. Revue Élev. Méd. vét. Pays trop., 1986, 39 (3-4):381-393.

6. HOOGSTRAAL (H.). African Ixodoidea. Ticks of the Sudan. Cairo, Egypt, U.S. Naval Med. Res. Unit, 1956, 3. 1101 p.

7. ILCA (International Livestock Centre for Africa). Livestock production in sub-humid zone of West Africa : A regional survey. Addis Ababa, ILCA, 1979. $184 \mathrm{p}$

8. IRZ (Institut de Recherches Zootechniques). Rapports annuels 1983, 1984. 1985. Yaoundé. IRZ.

9. MBAH (D.A.), Mortalités dues aux rickettsioses, trypanosomoses, piroplasmoses et streptotrichoses chez six génotypes de bovins à Wakwa, Cameroun, 1982. Revue Sci. Tech.. Yaoundé. 1982, 2 (2-3): 81-87.

10. MBITA MESSI (H.J.C.). Contribution à l'étude des types chorologiques de quelques espèces de la région de Yaoundé. Mém. maîtr. Botanique, Univ. Yaoundé, 1984.

11. MERLIN (P.), TSANGUEU (P.), ROUSVOAL (D.). Dynamique saisonnière de l'infestation par les tiques (Ixodoidea) des bovins dans les hauts plateaux de l'Ouest du Cameroun. Revue Élev. Méd. vét. Pays trop., 1986. 39 (3-4) : 377-379.

12. MOHAMED (A.D.). Seasonal incidence of Ixodoidea ticks of cattle in Northern Nigeria. Bull. Hlth Prod. Afr., 1977, 25 (3) : 273 293.

13. MOREL (P.C.). Morphologie, biologie et rôle pathogène des tiques. Maisons-Alfort, IEMVT, 1976. 73 p.

14. MOREL (P.C.). Study on Erhiopian ticks (Acarida, Ixodoidea). Maisons-Alforl, IEMVT, 1980. 332 p.

15. MOREL (P.C.), MOUCHET (J.). Les tiques du Cameroun (Ixodidae et Argasidae). Annls Parasit. hum. comp., 1958, 33 (1-2) : 69111.

16. MOREL (P.C.), MOUCHET (J.). Les tiques du Cameroun (Ixodidae et Argasidae). Annls Parasit. hum. comp., 1965, 40 (4) : $477-$ 496. 


\section{P.H. Bayemi}

17. MSTAT. Statistical package, Michigan State University. Crop and soil sciences. Agricultural economics. Institute of International Agriculture, 1985.

18. PEGRAM (R.G.), HOOGSTRAAL (H.), WASSEF (H.Y.). Ticks (Acari : Ixodoidea) of Ethiopia. Distribution, ecology and host relationships of species infesting livestock. Bull. ent. Res., 1981, $71: 339-359$.

19. RAGEAU (J.). Ixodoidea du Cameroun. Bull. Soc. Path. exot., 1951, 44 (7-8) : 441-446.

20. RAGEAU (J.). Clé pour l'identification des tiques du Cameroun. Annls Parasit. hum. comp., 1953, 28 (5-6) : $399-441$.

21. STRICKLAND (R.K.), TOBERT (K.), GERRISH (R.R.), HOURRIGAN (J.L.), SCHUBERT (G.O.). Ticks of veterinary importance. US Dept. Agriculture, 1976, 485.

22. TRONCY (P.M.), ITARD (J.), MOREL (P.C.). Précis de parasitologie vétérinaire tropicale. Maisons-Alfort, IEMVT, Paris, Ministère de la Coopération, 1981 (Manuels et précis d'élevage, $\mathrm{n}^{\circ} 10$ ). 